\title{
The Problem of the Similarity of Objects in Numerical Taxonomy
}

\author{
By R. JIČÍN AND Z. VAŠÍǏCKK \\ Museum Trutnov, Trutnov, Czechoslovakia \\ (Accepted for publication I8 June I969) \\ SUMMARY
}

\begin{abstract}
The existing definitions of coefficients of association in numerical taxonomy are not sufficiently well based theoretically. The present paper aims to make more objective the definition of the coefficient of association. It proceeds from the fact that the main source of difficulty is the third value (NC) used in scoring characters, and eliminates it. An improved coefficient of association between objects is then defined as the mean value of the coefficients of association of the individual pairs of forms of objects that are constructed by the method proposed below.
\end{abstract}

For interpretation we use the example in Table A-Io from Sokal \& Sneath (I963 p. 30I) which we reproduce here as Table I.

For the cases of those objects (OTU) which are described by characters with only two values '+' and '- ' (in Table I objects B, D, F) we shall accept the definition of the coefficient of association of Sokal \& Michener (1958), the Simple Matching Coefficient :

$$
S(M, N)=m / n
$$

where $m$ is the number of characters, both positive and negative, in which objects $M$ and $N$ agree, while $n$ is the total number of characters (see Table 2). This definition has two advantages. Firstly it interprets the coefficient of association as a probability that the objects agree on a randomly chosen character of the set under study. This definition, unlike others, is thus not based on a merely empirical approach, but proceeds from a theoretical basis of probability theory. Secondly, the definition presupposes the same value for the positive or negative form of the characters. This is a conception which consistently respects Adanson's principle of equal weight for every character and which is also currently used in logic. Just as we cannot quote any objective criterion that would generally distinguish the importance of two randomly chosen characters, so we cannot quote a criterion that would generally distinguish whether the positive state of character, $(+Q)$ or its negation $(-Q)$ is of greater importance. We should thus try to apply Adanson's principle so that it does not matter which state we score ' + ' or ' - '.

We also try to respect this definition in those cases where the characters have, besides the value ' + ' and '-', yet a third value ' $N C$ ', expressing indefinite value. We assume that ' $\mathrm{NC}$ ' denotes those cases where $+\mathrm{Q}$ or its logical negation $-\mathrm{Q}$ cannot be assigned unambiguously. This means that our further consideration refers only to those cases where the value ' $\mathrm{NC}$ ' means that the object must in theory have the 
character $+Q$ or $-Q$, but where the assignment of $+Q$ or $-Q$ cannot for some reason be decided. We thus consider the value ' $\mathrm{NC}$ ' in the sense of 'missing data' (cf. Sokal \& Sneath, I963 pp. 162-163) and not in the sense of 'missing characters' or 'missing organs'. The value 'NC' in the last two cases can be eliminated by the syntactic method (see Jičín, Pilous \& Vašíček, 1969).

Table I. The examples of OTU's and characters from

Sokal \& Sneath (I963) used for illustration

\begin{tabular}{ccccccc}
\multirow{2}{*}{ Characters } & A & B & C & D & E & F \\
I & - & + & - & + & - & + \\
2 & - & + & - & + & - & + \\
3 & + & - & + & - & + & - \\
4 & + & - & + & - & + & + \\
5 & NC & + & - & + & Objects & - \\
6 & NC & + & NC & + & - & - \\
7 & + & - & + & - & + & + \\
8 & - & + & - & + & - & + \\
9 & - & + & - & + & - & - \\
IO & + & - & + & - & + & -
\end{tabular}

If we do not know whether 'object $M$ has the character $+\mathrm{Q}$ ' or 'object $M$ has the character $-\mathrm{Q}$ ' (the latter is equivalent to 'object $M$ has not the character $+\mathrm{Q}$ ') we simply conclude that 'object $M$ has the character $+\mathrm{Q}$ or $-\mathrm{Q}$ '. We thus interpret the value ' $\mathrm{NC}$ ' of the character $\mathrm{Q}$ as an alternative to the values ' + ' and ' - ' in the description of object $M$. If there is only this one character $\mathrm{Q}$ with the value ' $\mathrm{NC}$ ' we treat it as the description of two hypothetical forms, $M_{1}$ and $M_{2}$, of the object. These two forms agree in all characters except $\mathrm{Q}$. We therefore assume that object $M$ may at least in theory occur either in the form $M_{1}$ (with $+\mathrm{Q}$ ) or in the form $M_{2}$ (with $-\mathrm{Q}$ ). We can illustrate this by object $C$ (from Table I), whose character 6 has the value 'NC', as follows:

$\begin{array}{cccccc}\text { Characters } & C_{1} & C_{2} & \text { Characters } & C_{1} & C_{2} \\ \mathbf{I} & - & - & 6 & + & - \\ 2 & - & - & 7 & + & + \\ 3 & + & + & 8 & - & - \\ 4 & + & + & 9 & - & - \\ 5 & - & - & 10 & + & +\end{array}$

Generally, this means that the value ' $N C$ ' can be excluded from consideration in that an object with $p$ characters scored ' $\mathrm{NC}$ ' is now described by $2^{p}$ columns, expressing all the forms the object could adopt.

Let us now establish the coefficient of association between object $C$ and, let us say, object $F$ (from Table I). If we determine the values of the coefficient of association, we find for $F$ and $C_{1}$ that $S\left(F, C_{1}\right)=0.5$. For $F$ and $C_{2}, S\left(F, C_{2}\right)=0.6$. We assume that object $C$ adopts both forms $C_{1}$ and $C_{2}$ with the same probability (we know nothing about the likelihood that character 6 in object $C$ is positive or negative and cannot prefer one over the other). We must therefore take the resultant coefficient of associa- 
tion as the mean value of $S\left(F, C_{1}\right)$ and $S\left(F, C_{2}\right)$. The new coefficient of association is then

$$
S(F, C)=\frac{0.6+0.5}{2}=0.55
$$

Generally, this means that if object $M$ has $p$ characters with the value 'NC' (described by $2^{p}$ columns, so that it may appear in the forms $M_{1}, M_{2}, \ldots, M_{2^{p}}$ ) and if object $N$ has $q$ characters with the value NC (occurring in $2^{q}$ forms, $N_{1}, N_{2}, \ldots$, $N_{2^{q}}$ ), our suggested coefficient of association $S(M, N)$ is given by the mean value of all the coefficients of association $S\left(M_{i}, N_{j}\right)$, where $M_{i}$ is any one of the forms $M_{1}, M_{2}$, $\ldots, M_{2^{p}}$, and $N_{j}$ any one of the forms $N_{1}, N_{2}, \ldots, N_{2^{q}}$. Hence:

$$
S(M, N)=\frac{\sum_{i=1}^{2^{p}} \sum_{j=1}^{2^{q}} S\left(M_{i}, N_{j}\right)}{2^{p+q}} .
$$

We can simplify equation (2) to the form

$$
S(M, N)=\frac{m+r / 2}{n},
$$

where $m$ is the number of agreements between the ' + ' and ' - ' values of objects $M$ and $N, r$ the sum of agreements and disagreements involving the 'NC' values, and $n$ the total number of characters (see Table 2). This definition permits us to calculate the coefficient of association without having to construct all the $2^{p}$ and $2^{q}$ alternatives. Hence it is of importance especially for those cases where there are many ' $\mathrm{NC}$ ' values.

Table 2. The possible combinations of character states on comparing objects $M$ and $N$

\begin{tabular}{cccc} 
& \multicolumn{3}{c}{ Object $M$} \\
\cline { 2 - 4 } Object N & $(+)$ & $(-)$ & $\mathrm{NC}$ \\
+ & $n_{+,+}$ & $n_{+,-}$ & $n_{+, \mathrm{NO}}$ \\
- & $n_{-,+}$ & $n_{-,-}$ & $n_{-, \mathrm{NC}}$ \\
$\mathrm{NC}$ & $n_{\mathrm{NC},+}$ & $n_{\mathrm{NC},-}$ & $n_{\mathrm{NC}, \mathrm{NC}}$
\end{tabular}

$n_{+,+}=$the number of character in which both objects are positive ( $c f$. Sokal \& Sneath, 1963

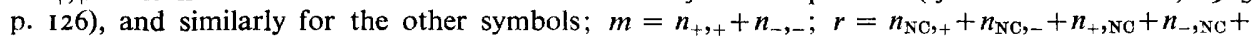
$n_{\mathrm{NO}, \mathrm{NC}} ; n=r+n_{+,+}+n_{+,-}+n_{-,+}+n_{-,-}$.

In Table 3 are presented all the similarities for all objects and their hypothetical forms, respectively, while Table 4 shows the mutual similarity of the objects using equation 3 .

This Table can be compared with the results of other formulae for coefficients of association, as given by Sokal \& Sneath (1963, p. 304) for these examples (Table 5).

This comparison shows that the new definition expresses more adequately the relationship between the pairs of objects. For example, of the previous ones, only the coefficient $S^{R R}$ distinguishes between $S(B, D)$ and $S(C, E)$, although in the first case identical objects are involved, while in the second case evidently not. It also makes a finer distinction in the similarities of the individual pairs (thus according to the 
existing formulae there are only 4 or 5 different $S$ values, but we obtain 7 different values according to the new formula). The fundamental difference resides, of course, in the fact that the new definition employs the value ' $\mathrm{NC}$ '. Let us consider the extreme case where for the objects $M, N$ and the characters I to Io all values are ' $\mathrm{NC}$ '. This means that we do not know anything at all about the assignment of any character

Table 3. The similarity values between all objects in Table $I$ and the hypothetical forms of them, using equation I

$\begin{array}{cccccccccccc}\text { Objects } & A_{1} & A_{2} & A_{3} & A_{4} & B & C_{1} & C_{2} & D & E_{1} & E_{2} & F \\ A_{1} & - & - & - & - & 0.2 & 0.9 & 0.8 & 0.2 & 0.9 & 0.8 & 0.4 \\ A_{2} & - & - & - & - & 0 . \mathrm{I} & 0.8 & 0.9 & 0 . \mathrm{I} & \mathrm{I} \cdot 0 & 0.9 & 0.5 \\ A_{3} & - & - & - & - & 0 . \mathrm{I} & \mathrm{I} \cdot 0 & 0.9 & 0 . \mathrm{I} & 0.8 & 0.9 & 0.5 \\ A_{4} & - & - & - & - & 0.0 & 0.9 & \mathrm{I} \cdot 0 & 0.0 & 0.9 & 1 \cdot 0 & 0.6 \\ B & - & - & - & - & \mathrm{I} \cdot 0 & 0 . \mathrm{I} & 0.0 & \mathrm{I} \cdot 0 & 0 \cdot \mathrm{I} & 0.0 & 0.4 \\ C_{1} & - & - & - & - & - & - & - & 0.1 & 0.8 & 0.9 & 0.5 \\ C_{2} & - & - & - & - & - & - & - & 0.0 & 0.9 & \mathrm{I} \cdot 0 & 0.6 \\ D & - & - & - & - & - & - & - & 1 \cdot 0 & 0 . \mathrm{I} & 0.0 & 0.4 \\ E_{1} & - & - & - & - & - & - & - & - & - & - & 0.5 \\ E_{2} & - & - & - & - & - & - & - & - & - & - & 0.6 \\ F & - & - & - & - & - & - & - & - & - & - & 1.0\end{array}$

Table 4. The similarity values between the objects in Table I using equation 3

\begin{tabular}{|c|c|c|c|c|c|c|}
\hline \multirow[b]{2}{*}{ Objects } & \multicolumn{6}{|c|}{ Objects } \\
\hline & $A$ & $B$ & $C$ & $D$ & $E$ & $F$ \\
\hline$A$ & 0.9 & 0.1 & 0.9 & $0 \cdot \mathrm{I}$ & 0.9 & 0.5 \\
\hline$B$ & $\ldots$ & $1 \cdot 0$ & 0.05 & $1 \cdot 0$ & 0.05 & 0.4 \\
\hline$C$ & - & - & 0.95 & 0.05 & 0.9 & 0.55 \\
\hline$D$ & - & - & - & $1.0^{\circ}$ & 0.05 & 0.4 \\
\hline$E$ & - & - & - & - & 0.95 & 0.55 \\
\hline$F$ & - & - & - & - & $\ldots$ & $1 \cdot 0$ \\
\hline
\end{tabular}

Table 5. Values of the other coefficients described by Sokal \& Sneath (I963)

Coeffi-

\begin{tabular}{|c|c|c|c|c|c|c|c|c|c|c|c|c|c|c|c|}
\hline $\mathrm{CI}$ & $B$ &,$C$ & $A, D$ & $1, E$ & ,F & $3, C$ & $D$ & $3, E$ & $B, F$ & $C, D$ & $C, E$ & $F$ & $D, E$ & $D, F$ & $E, F$ \\
\hline$S M$ & 0 & 0 & 0.0 & $I \cdot 0$ & 5 & 0.0 & I. & 0.0 & 0 & 0.0 & & & ח. & & -8 \\
\hline & & 1.0 & 0.0 & & 33 & 0.0 & & 0.0 & & & & & & & ?33 \\
\hline$R R$ & 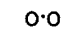 & 0.5 & . 0 & & .25 & 0.0 & 0 . & 0.0 & & & & & & & $\cdot 22$ \\
\hline$D$ & 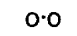 & .0 & $\cdot 0$ & & $\cdot 5$ & 0.0 & & 0.0 & & & & & & & \\
\hline 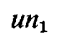 & 0 & $1 \cdot 0$ & 0.0 & 1 & 0.67 & 0.0 & & 0.0 & & & & & & & $\cdot 71$ \\
\hline$n_{2}$ & 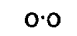 & $1 \cdot 0$ & 0.0 & 1 & 0.2 & 0.0 & & 0.0 & 0.1 & & I & & 0 & & $\cdot 2$ \\
\hline 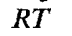 & 0 & .0 & .0 & 1.0 & $\cdot 33$ & 0.0 & $\mathbf{I}$ & 0.0 & 0.2 & & $I^{*}$ & & 0 & & -38 \\
\hline$K \mathrm{I}$ & & 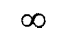 & 0 & $\infty$ & 5 & 0.0 & o & 0 & & & & & & & \\
\hline$\ell n_{\mathrm{S}}$ & & & 0 & $\infty$ & 1.0 & 0.0 & $\alpha$ & $\cdot 0$ & & 0 & $\infty$ & 5 & 0 & & $\cdot 2$ \\
\hline$K$ & & & 10 & & & 0.0 & & & & & & & & & 5 \\
\hline$u n_{4}$ & 0 & . 0 & 0.0 & 1.0 & 0.5 & $0 . \mathrm{C}$ & $1 \cdot$ & ० 0 & 2 & & {$[\cdot$} & 5 & o & & 5 \\
\hline . & & & 0.0 & I & 03 & 00 & & 0.0 & & & & & & & 5 \\
\hline$u n$ & & . & 0.0 & 10 & 0.25 & 0.0 & $1 \cdot 0$ & 0.0 & 0.17 & & & 3 & 0.0 & 17 & 3 \\
\hline$H$ & & $I \cdot O$ & $-I \cdot O$ & 1.0 & 0.0 & O० & $1 \cdot 0$ & $-I \cdot O$ & -0.2 & 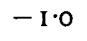 & & & 0 & 2 & $\cdot 1$ \\
\hline 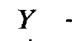 & & $I \cdot O$ & $-I \cdot O$ & $I \cdot 0$ & 0.0 & $-I \cdot O$ & $I \cdot O$ & $-I \cdot O$ & -0 & $-I \cdot O$ & 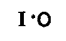 & 0.2 & $I \cdot O$ & & $D \cdot 2$ \\
\hline 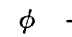 & $-I \cdot 0$ & 1.0 & $-I \cdot O$ & 1.0 & 0.0 & $-I \cdot 0$ & $1 \cdot 0$ & $-I \cdot 0$ & -0.17 & $-I \cdot O$ & I.O & 0.1 & $-I \cdot O$ & -0.17 & D. I \\
\hline
\end{tabular}


state to the two objects. With most of the existing definitions, $S(M, N)=0$. This result, however, is not logical, because the finding of complete dissimilarity requires a decision on the assignment of character states to the objects. If we do not know anything about this we ought to assume for each character that the objects $M$ and $N$ may agree or disagree with the same probability and that, therefore, $S(M, N)$ must equal $\frac{1}{2}$. This is the actual result obtained by the new definition. We may also note, though this ordinarily has little practical importance, that comparison of an object with itself yields a value less than $\mathrm{I}$ if it has characters scored ' $\mathrm{NC}$ ', and this can be seen in Table 4. This is an expression of the uncertainty about the states of these characters.

The authors are obliged to Dr O. Lysenko, CSc, Entomologický Ústav ČSAV, Praha, for revision and correction of the manuscript of this paper.

\section{REFERENCES}

Jičín, R., PIlous, Z. \& VAŠí̌̌eK, Z. (1969). Grundlagen einer formalen Methode zur Konstruktion und Bewertung von Bestimmungsschlüsseln. Preslia 41, 7 I.

SOKAL, R. R. \& MiCHENER, C. D. (I958). A statistical method for evaluating systematic relationships. Kans. Univ. Sci. Bull. 38, I409.

Sokal, R. R. \& Sneath, P. H. A. (1963). Principles of Numerical Taxonomy. San Francisco and London: Freeman. 